\title{
RECENT ABSOLUTE CALIBRATION WORK AT PALOMAR MOUNTAIN
}

\author{
J. B. OKE and R. SCHILD \\ Mount Wilson and Palomar Observatories, Carnegie Institution of Washington, \\ and California Institute of Technology, U.S.A.
}

\begin{abstract}
A four inch hermetically sealed reflecting telescope has been attached to the prime-focus scanner to provide an observing system for absolute calibration work. Light sources, including a platinum and a copper furnace, are observed at a distance of approximately 1200 feet. Since the telescope focal length is only 13 inches these sources look like stars. Thus bright stars such as $\alpha$ Lyr are compared directly with the light sources under identical instrumental conditions.

It has been found that horizontal extinction can be abnormally large; therefore it is measured several times each night. The absolute calibration is being derived at present from $3500 \AA$ to $11000 \AA$ and will be extended to $3200 \AA$ as soon as possible. The calibration lies approximately midway between that measured recently by Hayes and that adopted some years ago by Oke. A very preliminary value for the absolute flux from $\alpha \mathrm{Lyr}$ at $5556 \AA$ is $3.5 \times 10^{-20} \mathrm{ergs} \mathrm{sec}^{-1} \mathrm{~cm}^{-2} \mathrm{~Hz}^{-1}$ or $3.4 \times 10^{-9} \mathrm{ergs}$ $\mathrm{sec}^{-1} \mathrm{~cm}^{-2} \AA^{-1}$.
\end{abstract}

\section{Introduction}

The largest inherent uncertainty in the measurement of absolute fluxes and spectral energy distributions of astronomical objects is the absolute calibration of the primary standard star, $\alpha$ Lyr. A summary of the early work, which was largely photographic, has been given by Code (1960). More recent photoelectric absolute calibration work has been reported by Bahner (1963), Wolff et al. (1968), Haritonov (1963) and Willstrop (1965). These photoelectric studies make use of calibrated ribbon filament lamps and they disagree with each other in some cases by as much as $10 \%$.

The present work was undertaken to try to improve the absolute calibration of $\alpha$ Lyr. To achieve the highest possible accuracy the following decisions were made: (1) The most stable possible photoelectric system, telescope, and spectrometer were built and used. (2) The equipment was installed at an excellent photometric site, namely Palomar Mountain. (3) Absolute black-body light sources were used directly, eliminating the need for calibrated strip lamps which must be calibrated against the black body sources.

\section{Light Sources}

Two light sources have been used. These are black bodies generated by either a platinum or copper furnace. In both cases the observed radiation comes from the base of a sight tube which is immersed in a crucible of metal. Care is taken to insure that radiation is received only from the bottom of the sight tube and not from the cooler walls. All observations are made during the stable temperature plateaux which occur while the platinum or copper is in the process of melting or freezing. Apart from small corrections for emissivity, etc., the radiation is that of a black body at the melting point of the metal being used in the crucible. The platinum is heated by means of a 10 
kilowatt RF induction generator which heats the platinum directly. The copper is heated by means of a simple electric furnace. Both the platinum and copper black body sources follow closely the designs of the National Bureau of Standards (Wensel et al., 1931; Lee, 1969).

The melting point of copper is very close to that of gold which provides the fundamental standard at high temperatures. The melting point is taken to be $1357.9 \mathrm{~K}$ which is on the new 1969 practical temperature scale which has been defined to agree as closely as possible with the thermodynamic temperature scale. Because the copper melting point is low, this source could only be observed from $6300 \AA$ to $11000 \AA$. The uncertainty in the temperature, which is much less than $1 \mathrm{~K}$, introduces uncertainties in the absolute fluxes in this wavelength range of only a few tenths of $1 \%$ i.e., negligible compared with most of the other errors.

The temperature of melting platinum on the new 1969 practical-temperature scale is quoted as $2045 \mathrm{~K}$. However, the uncertainty in this number is approximately $2^{\circ}$. The platinum black body is sufficiently hot that it can be observed from $3400 \AA$ to $11000 \AA$ and therefore is also useful in the blue and violet.

\section{Observing Equipment}

Since both the furnaces and the star $\alpha$ Lyr have to be observed with the same equipment, it is necessary to have a suitable telescope. The telescope used is a 4 inch diameter $f / 3.3$ Newtonian reflector. The telescope has quartz windows and is hermetically sealed so that the mirrors do not become dusty or age appreciably. Since the focal length is only 13 inches, the black bodies, which are approximately 1200 feet from the telescope, look like star images. The light from the telescope is analyzed using the prime-focus scanner from the 200 -inch telescope, operated as a monochromator. The monochromatic output light is measured by photomultiplier tubes and high-speed pulse counting circuitry.

Since the spectrum scanner is a single-pass grating instrument great care must be taken to eliminate overlapping orders and scattered light. Various sets of glass filters are used for this purpose. Tests show that with the chosen filter combinations contamination is approximately $1 \%$ or less and suitable corrections can be made.

The telescope and spectrometer are mounted in the 18-inch dome at Palomar, using the 18-inch Schmidt telescope as a polar-axis mount. Observations can readily be made of both the light sources and bright stars such as $\alpha$ Lyr.

\section{Atmospheric Extinction}

The star or stars being observed are measured several times during the night at various zenith distances. These measures can be used to determine the atmospheric extinction at one wavelength relative to some standard wavelength with an accuracy of approximately $1 \%$. The absolute extinction at say $5556 \AA$, is somewhat more difficult to determine. 
A much more serious problem is the horizontal extinction between the light source and the telescope. If the atmosphere is homogeneous the horizontal extinction is $\frac{1}{20}$ of the vertical extinction and is never more than a few per cent. Unfortunately there can sometimes be haze near the ground which increases the horizontal extinction by large amounts but has practically no effect on vertical extinction. During each night when observations were being obtained an attempt was made to measure horizontal extinction by observing a quartz iodide lamp mounted first near the light source, and then on a tower half way between the telescope and light source. This technique has been most useful in detecting changes in the extinction, but also shows that the extinction on very good nights is not abnormal. Results given below were obtained only on the clearest possible nights.

\section{Observations}

On any given night observations were made either with a blue sensitive photomultiplier tube from $3500 \AA$ to $6100 \AA$ or with a red tube from $5800 \AA$ to $11000 \AA$. The band passes were $25 \AA$ and $100 \AA$ respectively for the blue and red observations. The furnace being used was run almost all night and observations were made on a great

TABLE I

Observational data

\begin{tabular}{|c|c|c|c|}
\hline Date (1969) & Wavelength range & Exit slit & Furnace \\
\hline Apr & 58 & 100 & \\
\hline & 5840 & 100 & latint \\
\hline 23 & $3500 \AA-6100 \AA$ & 25 & Platir \\
\hline May 24-25 & $3500 \AA-6100 \AA$ & $25 \AA$ & Platinum \\
\hline
\end{tabular}

many plateaux. All wavelengths being used could normally be measured at least once during a melt-plus-freeze cycle. Interspersed among the furnace measurements were observations of $\alpha$ Lyr and the quartz iodide lamp being used to measure extinction. The results given here are based on observations outlined in Table I. Observations had been made on many nights prior to those listed, to improve techniques.

\section{Results}

As indicated above the copper furnace provides an excellent radiation source for wavelengths above $6300 \AA$. The uncertainty in the platinum point is at least $2 \mathrm{~K}$ and there are further uncertainties caused by the geometry of the furnace. The actual radiating temperature of the platinum furnace can be estimated in two ways: (1) The absolute fluxes from the copper and platinum furnaces can be measured and compared at some specific wavelength which in our case is $6370 \AA$. This provides essentially a photo-electric pyrometer measurement. (2) The relative colors of the two sources can be compared over the wavelength range from $6370 \AA$ to $11000 \AA$. The former measure- 
ment is the more sensitive of the two. Both (1) and (2) indicate that the radiating temperature at the platinum point is $2038 \mathrm{~K}$ with an uncertainty of approximately $3 \mathrm{~K}$.

This low temperature is partially due to the fact that the sight tube does not provide a true thermodynamic enclosure because (a) there is an aperture to the outside world and (b) the upper part of the sight tube is at a lower temperature than the platinum point. De Vos (1954) has calculated the effects of using a cylindrical sight tube open at one end. Using a depth to radius value of 15 , the effective emissivity is 0.995 for diffuse reflection. This leads to a temperature correction of nearly $3 \mathrm{~K}$. In fact, the geometry

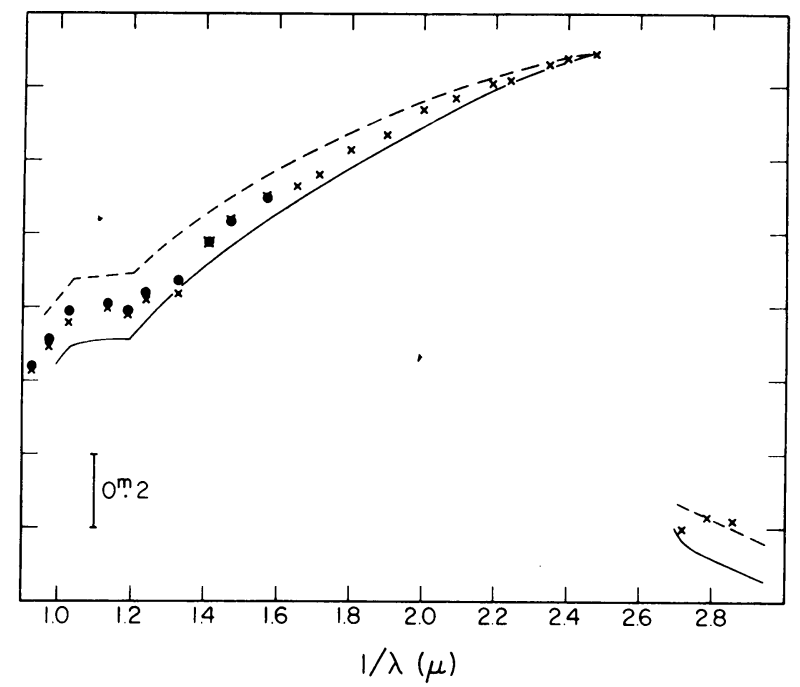

Fig. 1. The absolute spectral energy distribution of $\alpha$ Lyr. The ordinate is in magnitudes or $-2.5 \log f_{v}+$ const. Results from the copper furnace are shown by solid dots and those from the platinum furnace by crosses. The calibration of Hayes is shown by the solid curve, that adopted by Oke in 1964 by the broken curve.

used in the present furnace is somewhat different from that for which the calculations were made, and the thorium oxide sight tube may now be strictly a diffuse reflector. But, since the corrections appear to be of approximately the right size, the experimentally derived radiating temperature of $2038 \mathrm{~K}$ at the platinum point is adopted.

Preliminary results are shown in Figure 1. Here the absolute flux in terms of magnitudes $\left(-2.5 \log f_{v}\right)$ where $f_{v}$ is the flux in ergs $\sec ^{-1} \mathrm{~cm}^{-2} \mathrm{~Hz}^{-1}$ is plotted against $1 / \lambda$ where $\lambda$ is the wavelength in microns. The results from the copper and platinum furnaces are made to coincide at $1 / \lambda=1.54$. For comparison the calibration determined by Wolff et al. (1968) and that adopted by Oke (1964) are also shown. These have been normalized at $1 / \lambda=2.48$.

Our calibration is between that used by Oke and that of Hayes. It should here be emphasized that these results are still preliminary, particularly below the Balmer jump, since (a) further observations will be made and (b) small corrections, totalling approximately 1 to $2 \%$ are not yet included. These results, particularly as they bear on the 
melting point of platinum and the Balmer jump, are being checked through observation of another standard source in the form of a tungsten ribbon lamp which has been calibrated at the United States National Bureau of Standards.

It is possible to make a preliminary estimate of the accuracy of the data. As noted above, the uncertainty in the melting point of copper produces no significant error from $6370 \AA$ to $11000 \AA$. Below $6370 \AA$, where the platinum calibration must be used, an error of $3 \mathrm{~K}$ in the adopted radiating temperature at the melting point introduces an error of $1.5 \%$ at $3500 \AA$ relative to $6370 \AA$ and $1 \%$ at $4167 \AA$ relative to $6370 \AA$. The measurements themselves have an intrinsic inaccuracy, when finally averaged, of less than $1 \%$ and the atmospheric extinction for $\alpha$ Lyr is uncertain by approximately $1 \%$ above $4000 \AA$ and $2 \%$ below this wavelength. The horizontal extinction on the nights actually used for calibration should be accurate to better than $1 \%$. Combining the above errors suggests that the relative fluxes from $4036 \AA$ to $11000 \AA$ are not in error by more than $3 \%$, below $4036 \AA$ the uncertainty is approximately $4 \%$.

The fluxes for $\alpha$ Lyr shown in Figure 1 are all relative to some wavelength. To obtain a true absolute flux it is necessary to specify the absolute flux at some particular wavelength. The furnace sources are ideal for this since they are in fact very nearly black bodies. Using the copper furnace results we find for $\alpha$ Lyr at a wavelength of $5556 \AA(1 / \lambda=1.80)$ a preliminary flux of $3.5 \times 10^{-20} \mathrm{ergs} \mathrm{sec}^{-1} \mathrm{~cm}^{-2} \mathrm{~Hz}^{-1}$ or $3.4 \times$ $10^{-9}$ ergs sec ${ }^{-1} \mathrm{~cm}^{-2} \AA^{-1}$. The uncertainty is approximately $3 \%$. This differs markedly from the corresponding value of $3.8 \times 10^{-9} \mathrm{ergs} \mathrm{sec}^{-1} \mathrm{~cm}^{-2} \AA^{-1}$ adopted by Code (1960) and the value of $3.7 \times 10^{-9} \mathrm{ergs} \mathrm{sec}^{-1} \mathrm{~cm}^{-2} \AA^{-1}$ given by Willstrop (1965).

\section{Acknowledgement}

This work was supported by the Office of Naval Research through Contract No. N00014-67-A-0094-0005.

\section{References}

Bahner, K.: 1963, Astrophys. J. 138, 1314.

Code, A. D.: 1960, in Stars and Stellar Systems, Vol. VI, Stellar Atmospheres (ed. by J. L. Greenstein). University of Chicago Press, Chicago, Chap. 2, p. 50.

De Vos, J. C.: 1954, Physica 20, 669.

Haritonov, A. V.: 1963, Astron. Zh. 40, 339 (Soviet Astron. 7, 258).

Lee, R. D.: 1969, preprint.

Oke, J. B.: 1964, Astrophys. J. 140, 689.

Wensel, H. T., Roeser, W. F., Barbrow, L. E., and Caldwell, F. R.: 1931, N.B.S. J. Res. 6, 1103.

Willstrop, R. V.: 1965, Mem. Roy. Astron. Soc. 69, 83.

Wolff, S. C., Kuhi, L. V., and Hayes, D.: 1968, Astrophys. J. 152, 871.

\section{Discussion}

Morton: It is important to realize how important this absolute visual calibration is for interpretation of the ultraviolet data. We must know the energy corresponding to a particular apparent visual magnitude to obtain the observed ratio of ultraviolet to visual fluxes for comparing the observations with the model atmospheres. 